glauben Menschen, dass es sich bei den stereotypen Wahrnehmungen um objektives, empirisches Wissen handle (cf. Florack 2007, 143; Hahn/Hahn 2002, 41). (2) Ferner dienen Stereotype dazu, das Bild, das eine Person oder eine Gruppe von der Welt hat, aufrechtzuerhalten sowie Traditionen zu rechtfertigen. Ebenso können mit Stereotypen die Meinungen und Informationen von Anderen, die mit den eigenen Standpunkten nicht zusammenpassen, abgewertet, ignoriert oder sogar schlechtgemacht werden. (3) Drittens fungieren Stereotype identitätsstiftend für Gruppen. Über die Aktivierung von Stereotypen lassen sich soziale Gruppen (wie Familie, Peer-Gruppe, Vereine, religiöse Gruppen) leichter voneinander abgrenzen (,wir' versus ,die Anderen') und Auto- und Heterostereotype sowie Metastereotype (i. e. was ich denke, was der/die Andere über mich denkt) künstlich entwerfen. Es entstehen sogenannte ,imaginierte Gemeinschaften', die nach innen integrieren und nach außen abgrenzen. (4) Die Bestimmung des Anderen erfolgt dabei immer über das Eigene (das ,Wir'), wobei die Autostereotype tendenziell positiver angenommen werden als die Heterostereotype, das heißt, im Vergleich mit dem Anderen wird das Eigene tendenziell immer aufgewertet und das Andere abgewertet. (5) Als letzte Funktion von Stereotypen wird die politische Funktion angeführt, die oft auch im Zusammenhang mit propagandistischer oder ideologischer Funktion Erwähnung findet.

\title{
2.5 ZUSAMMENFASSUNG
}

Wie aus den vorangegangenen Abschnitten hervorgeht, handelt es sich bei Erzählungen, Medien und Stereotypen um drei Komponenten, mit denen Wissen und Welten konstruiert werden, wobei sich diese Komponenten in ihren Funktionen überlagern und gegenseitig verstärken. Eine narrative, mediale und stereotype Wissens- und Welterzeugung ist dem Forschungsgegenstand der Moralischen Wochenschriften ebenfalls insofern inhärent, als die Wochenschriftenproduzent*innen selbst stets aufs Neue postulieren, die ,Wirklichkeit' in ihren Blättern so ,abzubilden' wie sie ,ist ${ }^{6}$. Mit ihren repetitiven und laufend weitergetragenen Inhalten sind die Moralischen Wochenschriften Speicher und kollektiver Referenzpunkt für das sich neu konstituierende Wissen der Zeit, das insbesondere in der Mitte des 18. Jahrhunderts von einem „Paradigmenwechsel $^{28}$ mit einer radikalen Umstellung der gesellschaftlichen Semantik“ (Ertler 2003b, 31) gekennzeichnet ist. In den Wochenschriften werden alte, vornehmlich kirchliche Weltentwürfe umgedeutet und neue, säkulare Konzepte erstellt. Einerseits wird in ihnen eine aufklärerische Welt der Toleranz und der Meinungsfreiheit eingeführt, und andererseits wird in ihnen eine Welt der Geschlechterdifferenz, -ordnung und -hierarchien propagiert. Die Verbreitung der (stereotypen) Ideen, Konzepte und Wertvorstellungen über die Geschlechter und ihr Verhältnis zueinander innerhalb des

28 Unter ,Paradigma“ versteht Thomas S. Kuhn (1988), ,allgemein anerkannte wissenschaftliche Leistungen“ (10), die neuartig genug sind, ,um eine beständige Gruppe von Anhängern anzuziehen, [...], und gleichzeitig [...] noch offen genug [sind], um der neuen Gruppe von Fachleuten alle möglichen ungelösten Probleme zu stellen“ (25). In dem Maße wie ein Paradigma also einen gemeinsamen Referenzrahmen bietet, stellt es auch neue Forschungsperspektiven bereit. 
Mediums der Moralischen Wochenschriften führt zu einer bürgerlichen Wissens- und Welterzeugung. Diese verdient gerade im Aufklärungszeitalter besondere Beachtung, da in diesem Jahrhundert wichtige gesamt- und innergesellschaftliche Veränderungen in Hinblick auf das Geschlechterverständnis und das Geschlechterverhältnis in ganz Europa zu beobachten sind, deren Auswirkungen bis heute spürbar sind. ${ }^{29}$

Ähnlich wie die Ausbildung der modernen Nation, die sich durch Unterstützung medialer Netzwerke wie der Tageszeitung als ,imagined community (Anderson 2005) begreifen kann, wird die Bildung eines geschlechtsspezifischen Gemeinschafts- und Differenzgefühls unter bürgerlichen Frauen und Männern durch das mediale Netzwerk der Moralischen Wochenschriften befördert. Über Erzählungen wird in den Periodika einerseits eine als homogen dargestellte bürgerliche Welt in Abgrenzung zu den Welten der oberen und der unteren Schichten entworfen, andererseits wird innerhalb dieser bürgerlichen Welt eine geschlechtsspezifische Abgrenzung von Frauen und Männern vorgenommen. Diese Abgrenzung wird, wie die nachfolgende Analyse zeigen wird, vorrangig anhand bestimmter visueller Eindrücke von geschlechtsspezifischen Unterschieden vorgenommen. Gleichzeitig werden den bürgerlichen Frauen und Männern bestimmte invariante geschlechterstereotype (Charakter-)Eigenschaften und Verhaltensweisen zugeordnet. Mit diesen geschlechtsspezifischen Zuschreibungen geht schließlich auch eine wertende Tendenz einher, da bestimmte Eigenschaften als erwünscht, andere als unerwünscht präsentiert werden, wobei zeitgleich davon ausgegangen wird, dass es jeder und jedem möglich sei, sich zu einer tugendhaften Person zu entwickeln. Als Anreiz für die Hinwendung zur Tugendhaftigkeit gilt das Streben nach Glück, denn - so die Aufklärer*innen - nur tugendhafte Individuen, also jene, die die gesellschaftlichen Werte, Normen und Praktiken einhielten, könnten wirklich glücklich werden.

Die deskriptiven Erzählungen über bürgerliche Frauen und Männer, mit denen geschlechtsspezifische bürgerliche Werte, Normen und Praktiken überliefert werden, funktionieren schließlich als normative und voraussagende Wirklichkeitserzählungen, denn die beschriebenen geschlechtsspezifisch differenzierten Handlungs- und Verhaltensvorschriften zielen darauf ab, von der zeitgenössischen (imaginierten) bürgerlichen Gemeinschaft der Wochenschriftenleser*innen nachgeahmt und umgesetzt $\mathrm{zu}$ werden. Bevor jedoch eine ersichtliche moralische Besserung der Leser*innenschaft stattfinden kann, muss sie gelehrt werden, die zu verwerfende Ist-Situation zu erkennen. Deshalb sind die Wochenschriftenproduzent*innen um die Vermittlung der (für die Zeit neuen) sozialen Praktiken des Beobachtens und des Beurteilens bemüht. Damit sich die Leser*innen einer Selbst- und Fremddisziplinierung unterwerfen können, um den bürgerlichen Soll-Zustand zu erreichen, ist es nämlich elementar, dass diese überhaupt erst einmal die bürgerlichen Tugenden und Laster erkennen lernen.

Bevor nun aber das im 18. Jahrhundert verhandelte Wissen über die Geschlechter und ihr Verhältnis zueinander vorgestellt (Kapitel 4) sowie seine Umsetzung in den Moralischen Wochenschriften anhand der Ausarbeitung der spectatorialen Geschlechterkonstruktionen gezeigt werden (Kapitel 5), folgt nun die Beschreibung des Korpus und der methodischen Herangehensweisen.

29 Zur Entwicklung des europäisch geprägten kulturellen Wissens über die Geschlechter siehe Kapitel 4 zur geschlechtsspezifischen Wissens- und Welterzeugung im 18. Jahrhundert. 